\title{
Risk factors associated with the death of patients hospitalized for juvenile systemic lupus erythematosus
}

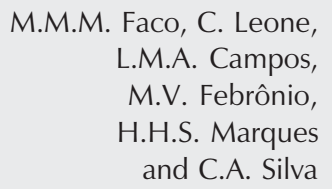

Unidades de Reumatologia e Infectologia Pediátricas, Instituto da Criança, Departamento de Pediatria, Faculdade de Medicina, Universidade de São Paulo, São Paulo, SP, Brasil

\section{Correspondence}

C.A. Silva

R. Senador Cesar L. Vergueiro, 494/82 05435-010 São Paulo, SP Brasil

Fax: +55-11-3069-8503

E-mail: clovisaas@icr.hcnet.usp.br

Research supported by a CNPq grant to C.A.A. Silva (No. 302469/2005-2).

Received February 15, 2006 Accepted March 20, 2007

\begin{abstract}
We assessed the risk factors associated with death in patients hospitalized for juvenile systemic lupus erythematosus (JSLE) and evaluated the autopsy reports. A total of 57,159 hospitalizations occurred in our institution from 1994 to 2003, 169 of them involving 71 patients with JSLE. The most recent hospitalization of these patients was evaluated. Patients were divided into two groups based on mortality during hospitalization: those who survived $(\mathrm{N}=53)$ and those who died $(\mathrm{N}=$ 18). The main causes of hospitalization were JSLE activity associated with infection in 52\% and isolated JSLE activity in 44\%. Univariate analysis showed that a greater risk of death was due to severe sepsis $(\mathrm{OR}=17.8, \mathrm{CI}=4.5-70.9)$, systemic lupus erythematosus disease activity index $(\mathrm{SLEDAI}) \geq 8(\mathrm{OR}=7.6, \mathrm{CI}=1.1-53.8)$, general infections $(\mathrm{OR}=6.1, \mathrm{CI}=1.5-25)$, fungal infections $(\mathrm{OR}=5.4, \mathrm{CI}=$ 3.2-9), acute renal failure ( $\mathrm{OR}=5.1, \mathrm{CI}=2.5-10.4)$, acute thrombocytopenia $(\mathrm{OR}=3.9, \mathrm{CI}=1.9-8.4)$, and bacterial infections $(\mathrm{OR}=2.3, \mathrm{CI}$ $=1.2-7.5)$. Stratified analysis showed that severe sepsis and SLEDAI $\geq 8$ were not confounder variables. In the multivariate analysis, logistic regression showed that the only independent variable in death prediction was severe sepsis $(\mathrm{OR}=98, \mathrm{CI}=16.3-586.2)$. Discordance between clinical diagnosis and autopsy was observed in 6/10 cases. Mortality of hospitalized JSLE patients was associated with severe sepsis. Autopsy was important to determine events not detected or doubtful in dead patients and should always be requested.
\end{abstract}

Key words

- Juvenile systemic lupus erythematosus

- Hospitalization

- Outcome

- Autopsy

- Severe sepsis

- Infection

\section{Introduction}

Juvenile systemic lupus erythematosus (JSLE) is a systemic autoimmune disease of unknown origin, which is more prevalent among females than males (5:1) (1). There are no data for the incidence of JSLE in Brazil.
JSLE prognosis is related to the affected organs/systems (2) and is worse for children and adolescents compared to adults, perhaps due to the higher rate of renal and neurological involvement attributed to JSLE (3). Over the last decades, the improvement of patient survival was mainly due to an early diagnosis and better therapeutic approach, espe- 
cially regarding the severe manifestations of the disease. These included the introduction of hemodialysis, renal transplantation and use of immunosuppressive drugs and their complications, i.e., use of potent antibiotic and hypertensive drugs (2).

Currently, the 5-year renal survival rate is $85-88 \%$ and the 10 -year survival rate is 60-65\% in the United States of America and Canada $(3,4)$. In Brazil, Appenzeller et al. (5) reported a 20-year JSLE survival of $48.1 \%$ at the Service of Pediatric Rheumatology, University of Campinas, Campinas, SP, Brazil.

Some investigators have described worse prognostic factors in JSLE such as male sex $(6,7)$, disease activity (8), active chronic disease (9), presence of cumulative disease damage $(10,11)$, arterial hypertension, proteinuria, acute renal failure (ARF) (4), lupus nephritis (12), neuropsychiatric involvement (13), antiphospholipid antibody syndrome (14), anti-DNA antibody increase (1), acute thrombocytopenia (10), infection (15), immunosuppressive treatment (10), and atherosclerosis (16). Most of the morbiditymortality studies on JSLE have evaluated worse prognostic factors and survival curves in patients from one or more Services of Pediatric Rheumatology, including outpatients and inpatients $(8,17)$.

Therefore, the scarcity of studies exclusively evaluating hospital admissions, deathassociated factors and autopsies in patients with JSLE, as well as the significant number of patients with this disease in our Service, stimulated the execution of the present study.

\section{Patients, Material and Methods}

During a 10-year period (January 1994December 2003) there were 57,159 hospital admissions at the Units of Instituto da Criança, São Paulo. Of these, 548 admissions ( $\mathrm{N}=$ 348 patients) were followed by the Pediatric Rheumatology Unit (mean of 4.6 admissions/month), with 169 of them (31\%) in- volving $71(20 \%)$ patients with JSLE. In the present study, we evaluated the most recent hospital admission of these 71 patients.

During the study period, 1907 deaths occurred in the Units of Instituto da Criança, and 18 of these $(0.94 \%)$ occurred in patients with JSLE. Autopsies were carried out in 10 of the 18 deaths (56\%) by the Discipline of Pathology of FMUSP, with previous authorization by the parents or persons responsible for the child or adolescent.

All patients met the classification criteria for JSLE according to the American College of Rheumatology (18) and were admitted to the infirmaries of Instituto da Criança (specialty infirmary, intensive and semi-intensive care units and emergency care) under the supervision of the Pediatric Rheumatology Unit. Patients who were admitted to receive drug therapy (methylprednisolone pulse therapy, cyclophosphamide pulse therapy and/or intravenous immunoglobulin) or to undergo diagnostic procedures such as digestive endoscopy and biopsies (kidney, skin, liver, and/or ganglion biopsies), among others, were excluded.

The study involved a retrospective evaluation of the medical files regarding demographic data, clinical characteristics of the disease, cause of hospital admission (infection, disease activity or others), seasonal variations of the admissions, and laboratory assessment performed during hospitalization. Additionally, the activity pattern, cumulative damage and course of the disease until hospital admission were analyzed, as well as the therapy used for the treatment of JSLE.

According to the most recent hospitalization, the patients were divided into two groups: survivors and deceased. The variables analyzed and compared in the two groups were: demographic data (age at onset, time of duration of disease and age at the last admission), gender, weight and height at admission, and total number and seasonality of admissions. 
The following characteristics of JSLE present at hospital admission were evaluated: arterial hypertension (19), ARF (20), renal involvement (presence of hematuria $>5,000 / \mathrm{mL}$, cylindruria $>3$ crosses in urinary sediment, and/or 24-h proteinuria $>500$ $\mathrm{mg} /$ day), lupus nephritis defined by the criteria of renal histology of the World Health Organization (6), neuropsychiatric involvement (21), antiphospholipid antibody syndrome (14), and bacterial, fungal or viral infections (presence of clinical syndrome associated with a high probability of infection and/or confirmation by radiological, microbiological or autopsy findings) (22).

The following characteristics present at hospital admission were also evaluated: acute thrombocytopenia (platelet number $<100,000 /$ $\mathrm{mm}^{3}$ within up to 1 month), 24-h proteinuria $(>0.5 \mathrm{~g} /$ day), nephritic proteinuria $(>50 \mathrm{mg}$ $\mathrm{kg}^{-1}$ day $\left.^{-1}\right)$, presence of anti-dsDNA antibody (evaluated by indirect immunofluorescence using Crithidia luciliae as substrate), and the presence of antiphospholipid antibodies in at least two measurements: ACL antibody (determined by ELISA with a Hemagen anticardiolipin ${ }^{\circledR}$ commercial kit (Columbia, MO, USA), and AL antibody detected by three methods: activated partial thromboplastin time, dilute Russell viper venom test, and kaolin clotting time test (23).

JSLE activity was evaluated by the index validated for the pediatric age range and known as systemic lupus erythematosus disease activity index (SLEDAI) (24). The disease was considered to be active with SLEDAI values $>1$. In the present study, moderate disease activity was defined as SLEDAI $\geq 8$. JSLE cumulative damage was evaluated according to the Systemic Lupus International Collaborating Clinics/ACRDamage Index (SLICC/ACR-DI). This is a score that has been validated for JSLE and has been applied to patients with disease for $>6$ months $(10,11)$. A score of $\geq 1$ was considered to indicate some cumulative damage of the disease. SLEDAI and SLICC-ACR/
DI scores were determined retrospectively during the 10-year period studied and were evaluated at the time of most recent hospitalization.

The pattern of disease activity was classified into three types according to Barr et al. (25): remissive (characterized by disease with no activity for at least 1 year), remissiverecidivous (characterized by periods of disease activity intercalated with periods of inactive disease for 1 year as determined at two or more office visits), and active chronic disease (continued disease activity for at least 1 year).

Sepsis was defined as proven by positive culture or strongly suspected infection by evidence of an infectious source associated with two or more clinical conditions considered for the diagnosis of systemic inflammatory response syndrome: a) core temperature $>37.5^{\circ} \mathrm{C}$ or below $36^{\circ} \mathrm{C}$; b) heart rate $>150$ bpm; c) respiratory frequency $>50 \mathrm{mpm}$; d) leukocytosis $>12,000$ leukocytes $/ \mathrm{mm}^{3}$ or $<4,000$ leukocytes $/ \mathrm{mm}^{3}$, or the presence of rods $>10 \%$. Severe sepsis was defined as sepsis associated with organ dysfunction, hypoperfusion or arterial hypotension (systolic arterial pressure $<10$ th percentile for age) $(22,26)$. Intra-hospital infection was defined in this study as any infection acquired 48-72 h after hospital admission.

JSLE treatment consisted of corticosteroids administered to all children at the time of hospitalization. The use of other treatments such as the antimalarials chloroquine or hydroxychloroquine and immunosuppressive drugs such as intravenous pulse therapy with cyclophosphamide, azathioprine, cyclosporine, mycophenolate mofetil, and/or methotrexate varied according to the manifestations of the disease in each patient.

In the present study, the clinical diagnosis of death was determined on the basis of the medical records, death certificate or autopsy request form. Goldman's classification (27) was used to detect discordance between the clinical diagnosis of cause of 
death and the anatomopathological diagnosis made at autopsy. In this classification, the authors divide the diagnoses into primary causes related to the fundamental disease or immediate cause of death and secondary causes including previous conditions or causes that contributed to death. The previous knowledge of discordant data, found only at autopsy, could modify the therapeutic management and would influence its prognosis.

\section{Statistical analysis}

The seasonality of the hospitalizations was analyzed by the calculation of the hospitalization rate/month. This rate was obtained by dividing the number of hospitalizations for each patient (in each trimester and in each semester) by the duration of disease in months (within a 10-year period). For each trimester and semester, the median hospitalization rate/month of all 169 hospitalizations was obtained, followed by the KruskalWallis test for the comparison of the medians of the four trimesters and two semesters. The Mann-Whitney non-parametric test was used to compare the median hospitalization rates/month per semester of the year. The Student $t$-test was carried out to compare mean age at the onset of the disease, duration of the disease up to hospitalization, age at most recent hospitalization, and number of hospitalizations for the two groups assessed and the Fisher exact test was used to compare genders in these groups. The Fischer exact test and Mann-Whitney test were used to compare the two groups regarding the following variables: clinical characteristics of the disease, activity, activity pattern, complementary exams, cumulative damage, and JSLE treatment. The Epi-Info 2000 software (Atlanta, GA, USA) calculated the weight and height $Z$ scores of each patient according to the reference patterns from the National Center of Health Statistics (28). Later, the non-paired $t$-test was used to com- pare the $\mathrm{Z}$ score means for weight and height at the last hospitalization between the survival and deceased groups.

Stratified analysis was used to determine the colinearity between the variables that presented statistical significance at the univariate analysis, such as: severe sepsis and SLEDAI $\geq 8$, ARF and use of intravenous cyclophosphamide pulse therapy, severe sepsis and immunosuppressive drug use, as well as severe sepsis and acute thrombocytopenia. The Epi-Info 2000 software, version $6.04 \mathrm{~B}$, was used to calculate the adjusted odds ratio (AOR) by the Mantel-Haenszl test for each possible confounder variable studied; subsequently, AOR was compared to the odds ratio (OR) obtained in univariate analysis.

Multivariate analysis was used with backward stepwise logistic regression. The independent variables chosen to enter the multivariate analysis model were those that presented a level of statistical significance of $\leq 5 \%$ in the univariate analyses. Five variables were selected in this model: severe sepsis, ARF, thrombocytopenia, mean proteinuria, and fungal infection, due to their biological plausibility regarding a possible cause-effect relation, considering death as dependent variable. All tests were carried out with the level of significance set at 5\% (P $<0.05$ ), considering the $95 \%$ confidence interval $(95 \% \mathrm{CI})$.

\section{Results}

The main causes of the most recent hospitalization of patients with JSLE were isolate activity in $31(44 \%)$ patients, activity associated with infection in $37(52 \%)$, and isolated infection in $3(4 \%)$. The most common place of hospitalization was the specialty infirmary of Instituto da Criança (38\%). Of the 18 patients who died, 17 were admitted to the Intensive Care Unit.

No significant differences were observed in the medians of the hospitalization rate/ 
month between the four trimesters of the year $(P=0.278)$ or between the two semesters of the year $(\mathrm{P}=0.279)$, indicating that there was no seasonal variation of hospitalization during the 10 years studied.

Comparison of the means for the children who died and those who survived did not show any significant difference regarding age at the onset of the disease, duration of the disease at the time of hospital admission, age at last hospitalization, and number of hospitalizations. Similarly, no significant differences were observed regarding gender, activity, patterns of disease activity or cumulative disease damage between the groups (Table 1).

Thirty-five bacterial infections occurred in 33 patients. Of note, 16 bacterial infections were diagnosed only by clinical features, e.g., pneumonia, sinusitis, otitis, meningitis (13 in the survivor group and 3 in the deceased group) and 19 by culture ( 7 in the survivor group and 12 in the deceased group, Table 2). In fact, in the two groups as a whole, 19 bacteria were identified by culture, 7 fungi by culture or at autopsy, and 6 viruses and one protozoan by the polymerase chain reaction (Table 2). Staphylococcus aureus and Pseudomonas aeruginosa were more frequent in deceased JSLE patients than in survivors $(50$ vs $0 \%, \mathrm{P}=0.0001 ; 17$ vs $2 \%, \mathrm{P}=0.047$; Table 2). Seven patients with fungal infections presented severe sepsis with moderate disease activity (SLEDAI 28 ), had a central catheter and received treatment for JSLE with immunosuppressive drugs (methylprednisolone and intravenous cyclophosphamide pulse therapy). Severe sepsis occurred in 16 patients who died (intra-hospital infection being diagnosed in 12 of them).

Univariate analysis showed that the patients with JSLE who subsequently died presented a significantly higher frequency of severe sepsis, general infection (bacterial, fungal and viral infection), moderately active disease, ARF, acute thrombocytopenia, cyclosporine and methotrexate use, and bacterial infection compared to the survivors. Of note, mean proteinuria was higher among patients who subsequently died than among survivors $(2.23 \pm 0.62$ vs $0.72 \pm 0.15 \mathrm{~g} /$ day, $\mathrm{P}=0.0116$; Table 3).

Table 1. Demographic characteristics, patterns of disease activity and cumulative damage of patients who survived or died with juvenile systemic lupus erythematosus.

\begin{tabular}{lcc}
\hline Demographic characteristics & Survivors $(\mathrm{N}=53)$ & Deceased $(\mathrm{N}=18)$ \\
\hline $\begin{array}{l}\text { Age at onset of disease } \\
\text { (months) }\end{array}$ & $119.07 \pm 42.2(32-208)$ & $136.55 \pm 46.66(38-216)$ \\
Age at last hospitalization & $146.16 \pm 51.36(60-256)$ & $171.05 \pm 30.09(48-255)$ \\
$\quad$ (months) & & \\
Disease duration (months) & $27.16 \pm 32.53(1-120)$ & $34.50 \pm 30.09(1-105)$ \\
Number of hospitalizations & $2.11 \pm 1.55(1-8)$ & $3.16 \pm 2.43(1-10)$ \\
Males & $11(21 \%)$ & $2(11 \%)$ \\
Active disease (SLEDAI $\geq 1)$ & $50(94 \%)$ & $18(100 \%)$ \\
Active chronic disease & $31(58 \%)$ & $13(72 \%)$ \\
SLICC-ACR/DI $\geq 1$ & $10(19 \%)$ & $6(33 \%)$ \\
\hline
\end{tabular}

Data are reported as mean $\pm S D$, with range in parentheses or as number with percent in parentheses. SLEDAI = Systemic Lupus Erythematosus Disease Activity Index; SLICC/ACR-DI = Systemic Lupus International Collaborating Clinics/ACR-Damage Index. There were no statistical differences between groups. Age at onset, age at last hospitalization, disease duration, and number of hospitalizations were compared by the Fisher exact test. Gender, active disease, active chronic disease and SLICC/ACRDI were compared by the Student $t$-test.

Table 2. Bacteria, fungi, viruses, and protozoa identified by culture, polymerase chain reaction, or autopsy of patients who survived or died with juvenile systemic lupus erythematosus.

\begin{tabular}{|c|c|c|}
\hline Organisms & Survivors $(N=53)$ & Deceased $(N=18)$ \\
\hline \multicolumn{3}{|l|}{ Bacteria } \\
\hline Staphylococcus aureus & $0(0 \%)$ & $9(50 \%)^{\star}$ \\
\hline Pseudomonas aeruginosa & $1(2 \%)$ & $3(17 \%)^{*}$ \\
\hline Escherichia coli & $4(8 \%)$ & $0(0 \%)$ \\
\hline Corynebacterium sp & $1(2 \%)$ & $0(0 \%)$ \\
\hline Streptococcus pyogenes & $1(2 \%)$ & $0(0 \%)$ \\
\hline \multicolumn{3}{|l|}{ Fungi } \\
\hline Candida albicans & $0(0 \%)$ & $3(17 \%)^{*}$ \\
\hline Aspergillus fumigattus & $0(0 \%)$ & $3(17 \%)^{\star}$ \\
\hline Cryptococcus neoformans & $0(0 \%)$ & $1(6 \%)$ \\
\hline \multicolumn{3}{|l|}{ Viruses } \\
\hline Herpes zoster & $4(8 \%)$ & $1(6 \%)$ \\
\hline Epstein-Barr & $0(0 \%)$ & $1(6 \%)$ \\
\hline \multicolumn{3}{|l|}{ Protozoa } \\
\hline Toxoplasma gondii & $0(0 \%)$ & $1(6 \%)$ \\
\hline
\end{tabular}

Data are reported as number with percent in parentheses.

${ }^{*} \mathrm{P}<0.05$ compared to survivors (Fisher exact test). 
Interestingly, comparison of the mean $\mathrm{Z}$ scores for height showed a statistically significant difference between the groups. The patients who subsequently died showed low mean $\mathrm{Z}$ scores compared to the survivors $(1.7 \pm 1.8$ vs $0.7 \pm 1.53, \mathrm{P}=0.038)$. The same was not true regarding weight assessment. In the stratified analysis, the OR of severe sepsis when adjusted for SLEDAI $\geq 8$ changed from 17.8 to an AOR of 15.2 ( $\mathrm{P}<0.001$, $95 \% \mathrm{CI}=4-58)$. This small variation between the OR and AOR indicated the absence of a confounding effect between variables. Likewise, the observation of the confounding effect of the presence of ARF and the use of intravenous cyclophosphamide pulse therapy revealed that the OR of univariate analysis changed from 5.1 to an AOR of $4.1(\mathrm{P}<0.046,95 \% \mathrm{CI}=1.98-8.58)$, suggesting the absence of colinearity between these variables.

Moreover, the determination of the confounding effect of the presence of severe sepsis and the use of immunosuppressive drugs showed that the OR of severe sepsis obtained in univariate analysis changed from

Table 3. Clinical features, laboratory examinations and treatments of patients who survived or died with juvenile systemic lupus erythematosus, in univariate analysis.

\begin{tabular}{lcccc}
\hline $\begin{array}{l}\text { Clinical features, laboratory } \\
\text { examinations and treatments }\end{array}$ & $\begin{array}{c}\text { Survivors } \\
(\mathrm{N}=53)\end{array}$ & $\begin{array}{c}\text { Deceased } \\
(\mathrm{N}=18)\end{array}$ & OR & $95 \% \mathrm{Cl}$ \\
\hline Severe sepsis & $6(11 \%)$ & $16(89 \%)^{*}$ & 17.8 & $4.5-70.9$ \\
General infection & $24(45 \%)$ & $16(89 \%)^{*}$ & 6.1 & $1.5-25.0$ \\
SLEDAI $\geq 8$ & $32(60 \%)$ & $17(94 \%)^{*}$ & 7.633 & $1.1-53.8$ \\
Fungal infection & $0(0 \%)$ & $6(33 \%)^{*}$ & 5.4 & $3.2-9.0$ \\
Acute renal failure & $4(11 \%)$ & $10(55 \%)^{*}$ & 5.1 & $2.5-10.5$ \\
Acute thrombocytopenia & $7(13 \%)$ & $10(55 \%)^{*}$ & 3.97 & $1.9-8.4$ \\
Proteinuria (g/day, mean $\pm \mathrm{SD})$ & $0.72 \pm 0.15$ & $2.23 \pm 0.62^{*}$ & - & - \\
Cyclosporine & $1(2 \%)$ & $3(17 \%)^{*}$ & 3.4 & $1.6-6.9$ \\
Methotrexate & $4(7 \%)$ & $5(28 \%)^{*}$ & 3.4 & $1.2-7.5$ \\
Bacterial infection & $20(38 \%)$ & $13(72 \%)^{*}$ & 2.3 & $1.2-7.5$ \\
IVCYC & $19(34 \%)$ & $11(61 \%)$ & - & - \\
Antimalarial drugs & $8(15 \%)$ & $3(17 \%)$ & - & - \\
\hline
\end{tabular}

Data are reported as number with percent in parentheses unless indicated. General infection = bacterial, fungal and viral infection; SLEDAI = Systemic Lupus Erythematosus Disease Activity Index; IVCYC = intravenous pulse therapy with cyclophosphamide; $\mathrm{OR}=$ odds ratio; $\mathrm{Cl}=$ confidence interval.

${ }^{*} \mathrm{P}<0.05$ compared to survivors (Fisher exact test or Student $t$-test).
17.8 to an $\mathrm{AOR}$ of $13.3(\mathrm{P}<0.001,95 \% \mathrm{CI}=$ 3.8-46.4), showing a slight confounding effect between the variables considered. In contrast, the determination of the confounding effect of the presence of acute thrombocytopenia and severe sepsis showed that the OR obtained in univariate analysis decreased from 3.97 to an AOR of $1.76(\mathrm{P}=0.097$, $95 \% \mathrm{CI}=1.04-2.91)$. The differences between these values suggest that there was a confounding effect caused by the interrelations of these variables.

Five variables were included in the multiple logistic regression analysis (severe sepsis, acute renal failure, thrombocytopenia, mean proteinuria, and fungal infection). The number of survivors with severe sepsis was $6 / 53$ and the number of deceased patients with severe sepsis was 16/18. Remarkably, severe sepsis was the only independent variable that showed significance for predicting death $\left(\mathrm{OR}=98, \mathrm{R}^{2}\right.$ of the Nagelkerke $=$ $0.676,95 \% \mathrm{CI}=16.38-586.22, \mathrm{P}<0.001)$.

There were no changes in the mortality pattern during the 10 years studied; however, due to the small number of deaths/year it was impossible to carry out statistical analysis. Ten of the 18 patients who subsequently died underwent autopsy (Table 4). Of note, risk factors for death by JSLE were observed in all of them: disease activity (SLEDAI $\geq 8$ ) in 17 patients, severe sepsis in 16 (associated with activity and severe sepsis in 15 of them), acute thrombocytopenia in 10 , and ARF in 10 .

Discordance between clinical diagnosis and autopsy was observed in 6 of 10 cases, including three with fungal infections and one with tuberculosis (primary causes), and one with diffuse glomerular proliferative nephritis and two with atherosclerosis (secondary causes), diagnosed exclusively at autopsy. The fungal infections identified exclusively at autopsy were diffuse aspergillosis in two patients and fungal myocarditis by Candida albicans in another (Table 4). 


\section{Discussion}

A limitation of the design of this study was a possible bias of the sample studied since the patients were selected from a JSLE population which is preferentially followed on an outpatient basis. Thus, the total patient population of the present Service of Pediatric Rheumatology was not assessed longitudinally and the survival curve for these patients was not generated. In addition, the sampling can be biased since the patients admitted to the hospital are expected to present a more severe disease compared to those who are followed exclusively on an outpatient basis and never needed hospitalization.

According to the medical literature, death occurs in $6 \%$ of outpatients and $42.8 \%$ of inpatients with JSLE $(5,8,29,30)$. The demographic data evaluated were not associated with a higher risk of death. A prevalence of a female:male ratio of 4.5:1 was observed, as also reported for other series $(7,10,11,31)$.

During the 10-year study period, a seasonal distribution was not demonstrated when all 169 hospitalizations due to JSLE were assessed. Some studies have reported that ultraviolet radiation, which is more intense during summer, can be the cause of SLE activation, determining an increase in the number of hospitalizations at this time of the year (32).

Of note, infection is one of the main causes of death in patients with JSLE $(7,15$, $17,29,30)$ and an important cause of Intensive Care Unit admission (33), as observed in the present study. The increase of bacterial infections, in particular Staphylococcus aureus and Pseudomonas aeruginosa, among these JSLE patients may be due to the use of specific drugs (corticosteroids and immunosuppressive drugs), to disease activity (3436) and to the presence of innate immunological alterations, such as phagocyte dys-

Table 4. Mortality of 18 patients with juvenile systemic lupus erythematosus.

\begin{tabular}{|c|c|c|c|c|c|c|}
\hline Patients & $\begin{array}{l}\text { Current age } \\
\text { (months) }\end{array}$ & $\begin{array}{l}\text { Disease duration } \\
\text { (months) }\end{array}$ & Gender & $\begin{array}{l}\text { Clinical diagnosis } \\
\text { of death }\end{array}$ & Autopsy & $\begin{array}{l}\text { Goldman } \\
\text { classification }\end{array}$ \\
\hline 1 & 187 & 67.2 & $\mathrm{~F}$ & SS, CNSV, RD, AT & NA & NA \\
\hline 2 & 48 & 1 & $\mathrm{~F}$ & SS, RD, ARF, AT, PH & Sepsis, RD, PH & Concordance \\
\hline 3 & 151.2 & 43.2 & $\mathrm{~F}$ & SS, RD, ARF, AT, pleuritis & NA & NA \\
\hline 4 & 129.6 & 1 & $\mathrm{~F}$ & SS, RD, ARF, AT & Sepsis, RD & Concordance \\
\hline 5 & 171.6 & 21.6 & $\mathrm{~F}$ & $\begin{array}{l}\mathrm{SS}, \mathrm{RD}, \mathrm{ARF}, \mathrm{AT}, \mathrm{PH}, \\
\text { pericarditis }\end{array}$ & Sepsis, RD, PH, pericarditis & Concordance \\
\hline 6 & 183.6 & 4 & $\mathrm{~F}$ & SS, CNSV, RD, ARF, AT & NA & NA \\
\hline 7 & 151.2 & 1 & $\mathrm{~F}$ & $\begin{array}{l}\mathrm{SS}, \mathrm{CNSV}, \mathrm{RD}, \mathrm{ARF}, \mathrm{AT} \text {, } \\
\text { pericarditis, pleuritis }\end{array}$ & $\begin{array}{l}\text { Sepsis, RD, fungal myocarditis, } \\
\text { bacterial endocarditis }\end{array}$ & Discordance, primary \\
\hline 8 & 199.2 & 55.2 & M & SS, RD, ARF, AT & NA & NA \\
\hline 9 & 220.8 & 54 & $\mathrm{~F}$ & $\mathrm{SS}, \mathrm{RD}$, pericarditis & Sepsis, pericarditis, AP & Discordance, secondary \\
\hline 10 & 121.2 & 1 & $\mathrm{~F}$ & SS, RD, ARF, pericarditis & Sepsis, ASP & Discordance, primary \\
\hline 11 & 49.2 & 11 & M & SS & NA & NA \\
\hline 12 & 255 & 33.6 & $\mathrm{~F}$ & SS, CNSV & TB, AP & Discordance, primary \\
\hline 13 & 163.2 & 7 & $\mathrm{~F}$ & SS, PH & $\begin{array}{c}\text { Sepsis, } \mathrm{PH} \text {, diffuse proliferative } \\
\text { nephritis }\end{array}$ & Discordance, secondary \\
\hline 14 & 196.8 & 60 & $\mathrm{~F}$ & SS, APS, RD, ARF, AT & Sepsis, thrombosis & Concordance \\
\hline 15 & 226.8 & 44.4 & $\mathrm{~F}$ & SS, pericarditis & ASP & Discordance, primary \\
\hline 16 & 199.2 & 60 & $\mathrm{~F}$ & SS, C, RD, ARF & NA & NA \\
\hline 17 & 180 & 36 & $\mathrm{~F}$ & CNSV & NA & NA \\
\hline 18 & 190.8 & 105 & $\mathrm{~F}$ & Hemorrhage (AT) & NA & NA \\
\hline
\end{tabular}

$\mathrm{AT}=$ acute trombocytopenia; $\mathrm{AP}=$ atheroma plaques; $\mathrm{APS}=$ antiphospholipid syndrome; $\mathrm{ARF}=$ acute renal failure; $\mathrm{ASP}=$ aspergillosis; $\mathrm{C}=$ cryptococcosis; CNSV = central nervous system vasculitis; $\mathrm{NA}=$ not applicable; $\mathrm{PH}=$ pulmonary hemorrhage; $\mathrm{RD}=$ renal disease; $\mathrm{SS}=$ severe sepsis; TB = disseminated tuberculosis. 
function, lymphopenia, functional asplenia, and a decrease of cytokines, immunoglobulins and complement fractions (33).

Because patients with JSLE are immunocompromised, either due to the disease itself or to its treatment, they are prone to opportunistic infections, mainly fungal infections and tuberculosis, which are generally associated with more severe diagnostic difficulties by mimicking the clinical manifestations of the disease, as observed in the patients studied here (34). The investigation of fungal infections is always very important for patients with JSLE, especially for those being treated with cyclophosphamide and corticosteroid pulse therapy, implanted with iv catheters, undergoing mechanical ventilation and requiring multiple antibiotic therapy.

A relevant aspect of the present study was the higher involvement of stature, at the time of hospitalization, in patients who subsequently died compared to those who survived. Stature is an indicator of quality of life in a chronic disease. The patients who subsequently died were possibly chronically malnourished, with a higher involvement of stature in relation to weight. These patients presented more aggressive disease and were chronic corticosteroid users, with prednisone doses $>10 \mathrm{mg} /$ day, which invariably suppress growth. Nevertheless, in these cases proteinuria and ARF could, among other things, cause edema and overweight, which would explain the lack of statistical difference regarding weight at hospitalization between the two groups analyzed.

Furthermore, JSLE is a systemic disease with possible confounding effects (colinearity) between the several clinical, laboratory and therapeutic variables implicated in the increase of morbidity-mortality. Thus, stratified analysis was used in this study as a strategy for adjustment of the effect of the variables studied that showed statistical association with death by univariate analysis. Of note, only acute thrombocytopenia and severe sepsis showed colinearity. Therefore, severe sepsis more probably caused acute thrombocytopenia and increased the death risk in the patients of this study.

Remarkably, the multivariate analysis model showed that only severe sepsis was the independent variable associated with death, with an important OR of 98, i.e., severe sepsis is a high-power variable for subsequent death. Nevertheless, it presented a broad confidence interval (16.4 to 586), probably due to the small sample size of this study. Thus, the model supports the fact that the other factors associated with subsequent death determined by univariate analysis had no importance in the final prediction of death.

The definition of severe sepsis in children requires the presence of organ and system dysfunction; among them, we can cite the hematological system, which involves the thrombocytopenia factor $(22,26,37)$. In the present study, $85 \%$ of the deaths occurred in Intensive Care Units, with $89 \%$ of these patients presenting severe sepsis. The evaluation of the presence of infection at hospital admission translates only partially the occurrence of this condition since the cumulative sepsis rate increases during hospital admission due to hospital-acquired infections (38). In the present study, intrahospital infection, developing to severe sepsis, occurred in 12 of the 16 patients in the deceased group and in 5 of 6 patients who survived.

At our institution, the Hospital Infection Commission is very active regarding the prevention of infection, as well as in the individualized discussion of the best use of antimicrobial agents in the presence of multiresistant intra-hospital bacteria. Gladman (9) studied the presence of infection in hospitalized JSLE patients and observed that one-third of the patients analyzed acquired the infection during the hospital stay, with the duration of hospitalization being directly proportional to the frequency of intra-hospital infection. Therefore, severe sepsis due to intra-hospital-acquired infection is a risk fac- 
tor for subsequent death in JSLE patients.

Studies on the frequency of autopsy in rheumatologic diseases are usually conducted on the adult population. In the present study, autopsy consent was given by 10 families of the 18 patients who died. This considerable frequency reflects not only the academic concern of our institution regarding its role as a university hospital, but also the affective bond and proximity between the medical team and the families, who, despite the suffering caused by the death of a loved one, still allowed the procedure to be carried out.

Autopsies are important to determine events that have not been clarified or that were uncertain at the time of death. In this series, discordance between the clinical diagnosis and the autopsy results occurred in six cases. Three fungal infections and one case of miliary tuberculosis were identified exclusively at autopsy (primary causes). Of note, amphotericin B was empirically administered to 2 of the patients with fungal infection. In contrast, 1 patient with aspergillosis and another with miliary tuberculosis did not receive proper treatment for their respective infections. Interestingly, atheroma plaques were observed at autopsy in 2 cases, both associated with dyslipidemia, i.e., elevation of low-density cholesterol fraction and decrease of the high-density fraction, according to the literature (16). Neither patient had received antimalarial drugs. These drugs (chloroquine or hydroxychloroquine) reduce dyslipidemia in adults with SLE, and in the last 3 years have been routinely used in all cases with JSLE at the Unit of Pediatric Rheumatology of Instituto da Criança, HCFMUSP, regardless of the organs and systems involved (36).

Therefore, the main factor associated with death of inpatients with JSLE was severe sepsis. Thrombocytopenia may seem to be a spurious variable (mistaken as severe sepsis), but this is not the case for moderate disease activity, which is important for the worse evolution of JSLE, being independent of severe sepsis.

The frequency of deaths in JSLE patients admitted to our Hospital was $25 \%$. These patients had an increased risk for severe sepsis. Because disease manifestation may mimic signs and symptoms of infection, a prompt diagnosis may be difficult. Autopsy showed infectious disease or atherosclerosis not detected previously and was important to determine undetected or doubtful events in dead patients and should always be requested.

\section{Acknowledgments}

The authors wish to thank Dr. Crésio Romeu Pereira of Department of Pediatric, University of São Paulo, for helping with the stratified statistical analysis of this study and Dr. Alfio Rossi Jr. of Pediatric Infectology Unit, Department of Pediatric, University of São Paulo. The authors also wish to thank the pathologists of the Discipline of Pathology, FMUSP, who carried out the autopsies.

\section{References}

1. Donadio JV Jr, Hart GM, Bergstralh EJ, Holley KE. Prognostic determinants in lupus nephritis: a long-term clinicopathologic study. Lupus 1995; 4: 109-115.

2. Ward MM, Pyun E, Studenski S. Mortality risks associated with specific clinical manifestations of systemic lupus erythematosus. Arch Intern Med 1996; 156: 1337-1344.

3. Lehman TJA. Systemic lupus erythematosus in childhood and adolescence. In: Wallace DJ, Hahn BH (Editors), Dubois' lupus erythematosus. Baltimore: Williams \& Williams; 1997.
4. McCurdy DK, Lehman TJ, Bernstein B, Hanson V, King KK, Nadorra $\mathrm{R}$, et al. Lupus nephritis: prognostic factors in children. Pediatrics 1992; 89: 240-246.

5. Appenzeller S, Marini R, Costallat LT. Damage did not independently influence mortality in childhood systemic lupus erythematosus. Rheumatol Int 2005; 25: 619-624.

6. Niaudet P. Treatment of lupus nephritis in children. Pediatr Nephrol 2000; 14: 158-166

7. Hilário MOE, Len CA, Sato El, Terreri MT, Sacchetti S, Okuda EM, 
et al. Lupus eritematoso sistêmico juvenil: resultados do estudo multicêntrico nacional. Rev Bras Reumatol 2002; 42: 85-89.

8. Singh S, Devidayal, Kumar L, Joshi K. Mortality patterns in childhood lupus - 10 years' experience in a developing country. Clin Rheumatol 2002; 21: 462-465.

9. Gladman DD. Prognosis of systemic lupus erythematosus and factors that affect it. Curr Opin Rheumatol 1992; 4: 681-687.

10. Brunner HI, Silverman ED, To T, Bombardier C, Feldman BM. Risk factors for damage in childhood-onset systemic lupus erythematosus: cumulative disease activity and medication use predict disease damage. Arthritis Rheum 2002; 46: 436-444.

11. Ravelli A, Duarte-Salazar C, Buratti S, Reiff A, Bernstein B, Maldonado-Velazquez MR, et al. Assessment of damage in juvenile-onset systemic lupus erythematosus: a multicenter cohort study. Arthritis Rheum 2003; 49: 501-507.

12. Trager J, Ward MM. Mortality and causes of death in systemic lupus erythematosus. Curr Opin Rheumatol 2001; 13: 345-351.

13. Vyas S, Hidalgo G, Baqi N, Von GH, Singh A. Outcome in AfricanAmerican children of neuropsychiatric lupus and lupus nephritis. Pediatr Nephrol 2002; 17: 45-49.

14. Campos LM, Kiss MH, D'Amico EA, Silva CA. Antiphospholipid antibodies and antiphospholipid syndrome in 57 children and adolescents with systemic lupus erythematosus. Lupus 2003; 12: 820826.

15. Gonzalez B, Hernandez P, Olguin H, Miranda M, Lira L, Toso M, et al. Changes in the survival of patients with systemic lupus erythematosus in childhood: 30 years experience in Chile. Lupus 2005; 14: 918-923.

16. Hayata AL, Borba EF, Bonfa E, Kochen JA, Goldenstein-Schainberg C. The frequency of high/moderate lipoprotein risk factor for coronary artery disease is significant in juvenile-onset systemic lupus erythematosus. Lupus 2005; 14: 613-617.

17. Wang LC, Yang YH, Lu MY, Chiang BL. Retrospective analysis of mortality and morbidity of pediatric systemic lupus erythematosus in the past two decades. J Microbiol Immunol Infect 2003; 36: 203-208.

18. Hochberg MC. Updating the American College of Rheumatology revised criteria for the classification of systemic lupus erythematosus. Arthritis Rheum 1997; 40: 1725.

19. Anonymous. The fourth report on the diagnosis, evaluation, and treatment of high blood pressure in children and adolescents. Pediatrics 2004; 114: 555-576.

20. Schwartz GJ, Brion LP, Spitzer A. The use of plasma creatinine concentration for estimating glomerular filtration rate in infants, children, and adolescents. Pediatr Clin North Am 1987; 34: 571-590.

21. Anonymous. The American College of Rheumatology nomenclature and case definitions for neuropsychiatric lupus syndromes. Arthritis Rheum 1999; 42: 599-608.

22. Levy MM, Fink MP, Marshall JC, Abraham E, Angus D, Cook D, et al. 2001 SCCM/ESICM/ACCP/ATS/SIS International Sepsis Definitions Conference. Crit Care Med 2003; 31: 1250-1256.

23. Brandt JT, Barna LK, Triplett DA. Laboratory identification of lupus anticoagulants: results of the Second International Workshop for Identification of Lupus Anticoagulants. On behalf of the Subcommittee on Lupus Anticoagulants/Antiphospholipid Antibodies of the
ISTH. Thromb Haemost 1995; 74: 1597-1603.

24. Brunner HI, Feldman BM, Bombardier C, Silverman ED. Sensitivity of the Systemic Lupus Erythematosus Disease Activity Index, British Isles Lupus Assessment Group Index, and Systemic Lupus Activity Measure in the evaluation of clinical change in childhoodonset systemic lupus erythematosus. Arthritis Rheum 1999; 42: 1354-1360.

25. Barr SG, Zonana-Nacach A, Magder LS, Petri M. Patterns of disease activity in systemic lupus erythematosus. Arthritis Rheum 1999; 42: 2682-2688.

26. American College of Chest Physicians/Society of Critical Care Medicine Consensus Conference: definitions for sepsis and organ failure and guidelines for the use of innovative therapies in sepsis. Crit Care Med 1992; 20: 864-874.

27. Goldman L, Sayson R, Robbins S, Cohn LH, Bettmann M, Weisberg $\mathrm{M}$. The value of the autopsy in three medical eras. $N$ Engl $\mathrm{J}$ Med 1983; 308: 1000-1005.

28. Ogden CL, Kuczmarski RJ, Flegal KM, Mei Z, Guo S, Wei R, et al. Centers for Disease Control and Prevention 2000 growth charts for the United States: improvements to the 1977 National Center for Health Statistics version. Pediatrics 2002; 109: 45-60.

29. Jacobsen S, Petersen J, Ullman S, Junker P, Voss A, Rasmussen $\mathrm{JM}$, et al. Mortality and causes of death of 513 Danish patients with systemic lupus erythematosus. Scand J Rheumatol 1999; 28: 7580.

30. Doria A, laccarino L, Ghirardello A, Zampieri S, Arienti S, SarziPuttini $\mathrm{P}$, et al. Long-term prognosis and causes of death in systemic lupus erythematosus. Am J Med 2006; 119: 700-706.

31. Yu HH, Lee JH, Wang LC, Yang YH, Chiang BL. Neuropsychiatric manifestations in pediatric systemic lupus erythematosus: a 20-year study. Lupus 2006; 15: 651-657.

32. Wysebeek AJ, Block DA, Eries LE. Prevalence and expression of photosensitivity in systemic lupus erythematosus. Ann Rheum Dis 1989; 48: 461-463.

33. Raj R, Murin S, Matthay RA, Wiedemann HP. Systemic lupus erythematosus in the intensive care unit. Crit Care Clin 2002; 18: 781 803.

34. Pryor BD, Bologna SG, Kahl LE. Risk factors for serious infection during treatment with cyclophosphamide and high-dose corticosteroids for systemic lupus erythematosus. Arthritis Rheum 1996; 39: 1475-1482.

35. Marks SD, Sebire NJ, Pilkington C, Tullus K. Clinicopathological correlations of paediatric lupus nephritis. Pediatr Nephrol 2007; 22: 77-83.

36. Silva CA. Lúpus eritematoso sistêmico juvenil. Rev Bras Reumatol 2006; 46 (Suppl 2): 6-7.

37. Tran DD, Groeneveld AB, van der Meulen J, Nauta JJ, Strack van Schijndel RJ, Thijs LG. Age, chronic disease, sepsis, organ system failure, and mortality in a medical intensive care unit. Crit Care Med 1990; 18: 474-479.

38. Angus DC, Linde-Zwirble WT, Lidicker J, Clermont G, Carcillo J, Pinsky MR. Epidemiology of severe sepsis in the United States: analysis of incidence, outcome, and associated costs of care. Crit Care Med 2001; 29: 1303-1310. 